Supplement of Nat. Hazards Earth Syst. Sci., 19, 137-151, 2019

https://doi.org/10.5194/nhess-19-137-2019-supplement

(C) Author(s) 2019. This work is distributed under

the Creative Commons Attribution 4.0 License.

(c) (1)

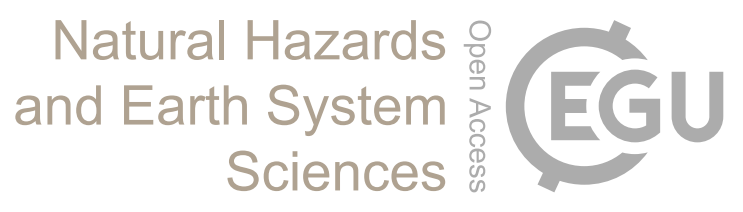

Supplement of

\title{
Economic damage and spillovers from a tropical cyclone
}

Manfred Lenzen et al.

Correspondence to: Arunima Malik (arunima.malik@sydney.edu.au)

The copyright of individual parts of the supplement might differ from the CC BY 4.0 License. 


\section{Table of contents}

SI 1: Sector classification used in this study

SI 2: Estimation of gamma matrix for Tropical Cyclone Debbie........................................... 3

SI 3: Raw data for production layer decomposition graphs................................................ 8

SI 4: Underlying raw data for main text's Figure 3............................................................... 9

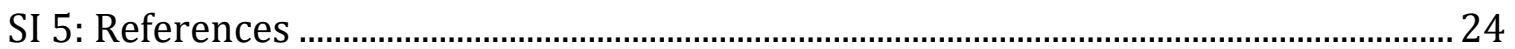




\section{SI 1: Sector classification used in this study}

1. Sheep

2. Grains

3. Beef cattle

4. Dairy cattle and pigs

5. Other agriculture

6. Sugar cane growing

7. Forestry and fishing

8. Coal, oil and gas

9. Non-ferrous metal ores

10. Other mining

11. Food manufacturing

12. Textiles, clothing and footwear

13. Wood and paper manufacturing

14. Chemicals, petroleum and coal products

15. Non-metallic mineral products

16. Metals, metal products

17. Machinery appliances and equipment

18. Miscellaneous manufacturing

19. Electricity supply, gas and water

20. Residential building construction

21. Other construction

22. Trade

23. Accommodation, cafes and restaurants

24. Road transport

25. Rail and pipeline transport

26. Other transport

27. Communication services

28. Finance, property and business services

29. Ownership of dwellings

30. Government administration and defence

31. Education

32. Health and community services

33. Cultural and recreational services

34. Personal and other services 


\section{SI 2: Estimation of gamma matrix for Tropical Cyclone Debbie}

Table SI 2.1 Total output (million dollars) by region and sector used for the estimation of the gamma matrix.

\begin{tabular}{|c|c|c|c|c|c|c|c|c|c|c|c|c|c|c|c|c|c|c|}
\hline & Rest of NSW & $\begin{array}{c}\text { NSW- } \\
\text { Richmond - } \\
\text { Tweed }\end{array}$ & VIC & $\begin{array}{l}\text { QLD- } \\
\text { Brisbane }\end{array}$ & $\begin{array}{c}\text { QLD-Wide- } \\
\text { Bay-Burnett }\end{array}$ & $\begin{array}{c}\text { QLD-Darling } \\
\text { Downs }\end{array}$ & $\begin{array}{l}\text { QLD-South } \\
\text { West }\end{array}$ & QLD-Fitzroy & $\begin{array}{c}\text { QLD-Central } \\
\text { West }\end{array}$ & QLD-Mackay & $\begin{array}{l}\text { QLD- } \\
\text { Northern }\end{array}$ & $\begin{array}{l}\text { QLD-Far } \\
\text { North }\end{array}$ & $\begin{array}{l}\text { QLD-North } \\
\text { West }\end{array}$ & SA & WA & TAS & АCT & NT \\
\hline 1 Sheep & 2054.87 & 0.46 & 1376.32 & 14.06 & 5.52 & 106.75 & 82.58 & 15.89 & 54.33 & 4.68 & 2.30 & 2.78 & 5.89 & 799.37 & 723.36 & 245.86 & 11.87 & 2.76 \\
\hline 2 Grains & 3964.44 & 15.99 & 2411.27 & 103.91 & 55.65 & 926.98 & 106.00 & 172.94 & 0.66 & 59.61 & 13.89 & 14.32 & 0.00 & 2352.62 & 2529.64 & 76.75 & 10.26 & 0.86 \\
\hline 3 Beef cattle & 2993.79 & 196.41 & 1746.66 & 355.23 & 402.54 & 559.63 & 308.44 & 482.30 & 232.72 & 208.82 & 123.09 & 150.51 & 219.55 & 720.94 & 922.96 & 257.34 & 10.02 & 170.01 \\
\hline 4 Dairy cattle and pigs & 988.77 & 83.81 & 3498.11 & 193.73 & 168.53 & 275.57 & 0.00 & 16.84 & 2.14 & 7.55 & 5.05 & 65.73 & 0.00 & 473.35 & 277.58 & 398.59 & 1.99 & 0.00 \\
\hline 5 other agriculture & 6051.67 & 605.33 & 5611.38 & 2441.55 & 1212.44 & 1022.14 & 178.84 & 270.50 & 53.87 & 237.31 & 491.51 & 1152.04 & 29.06 & 3397.03 & 2164.15 & 880.15 & 36.15 & 177.39 \\
\hline 6 Sugar cane growing & 39.48 & 61.91 & 0.00 & 40.99 & 191.40 & 0.00 & 0.00 & 1.84 & 0.00 & 381.60 & 421.93 & 251.86 & 0.00 & 0.00 & 0.00 & 0.00 & 0.00 & 0.92 \\
\hline 7 Forestry and fishing & 1586.50 & 111.29 & 1031.88 & 413.17 & 314.64 & 60.37 & 15.60 & 25.78 & 10.92 & 24.24 & 69.74 & 192.11 & 4.15 & 873.98 & 838.11 & 1427.63 & 22.94 & 132.25 \\
\hline 8 Coal, oil and gas & 29402.32 & 252.89 & 3818.91 & 13527.34 & 2060.75 & 2022.11 & 365.26 & 10706.70 & 93.35 & 15023.91 & 1279.20 & 323.30 & 28.85 & 3197.48 & 14439.27 & 229.45 & 23.76 & 255.75 \\
\hline 9 Non-ferrous metal ores & 3373.41 & 42.16 & 1682.25 & 1363.56 & 212.50 & 46.30 & 5.96 & 282.43 & 5.11 & 40.89 & 1337.47 & 1353.80 & 2649.15 & 2423.90 & 12129.58 & 794.17 & 11.07 & 1231.84 \\
\hline 10 Other mining & 4840.87 & 223.38 & 3196.15 & 3888.27 & 559.40 & 597.17 & 181.09 & 1066.26 & 20.23 & 1371.76 & 730.00 & 461.37 & 236.21 & 3146.09 & 34298.66 & 1018.39 & 54.99 & 586.65 \\
\hline 11 Food manufacturing & 23399.27 & 1191.76 & 24690.41 & 11498.44 & 1380.13 & 1578.69 & 115.30 & 951.83 & 27.27 & 923.98 & 1223.07 & 815.57 & 19.59 & 8876.13 & 5832.85 & 2622.63 & 276.18 & 191.90 \\
\hline 12 Textiles, clothing and footwear & 2901.07 & 85.88 & 3729.31 & 1138.76 & 55.38 & 85.69 & 5.90 & 26.08 & 0.00 & 31.02 & 53.58 & 39.75 & 3.98 & 589.59 & 745.88 & 160.04 & 39.49 & 32.67 \\
\hline 13 Wood and paper manufacturing & 6262.96 & 209.01 & 6480.85 & 2678.16 & 461.57 & 204.65 & 24.70 & 97.50 & 4.22 & 62.09 & 123.85 & 114.55 & 5.07 & 1930.12 & 1574.57 & 747.31 & 159.83 & 47.80 \\
\hline 14 Chemicals, petroleum and coal products & 22340.86 & 289.78 & 23559.36 & 10017.27 & 255.72 & 380.02 & 17.87 & 520.82 & 4.10 & 334.85 & 661.68 & 194.45 & 113.75 & 4209.49 & 7416.79 & 904.77 & 114.21 & 142.17 \\
\hline 15 Non-metallic mineral products & 5009.64 & 119.02 & 4018.35 & 2523.38 & 99.48 & 64.86 & 7.12 & 377.35 & 0.00 & 103.02 & 155.00 & 110.03 & 10.99 & 1268.93 & 1957.07 & 352.88 & 110.14 & 105.70 \\
\hline 16 Metals, metal products & 28323.36 & 419.46 & 23577.50 & 15238.29 & 745.68 & 884.81 & 60.26 & 3144.60 & 8.06 & 743.56 & 2041.71 & 1002.91 & 193.20 & 7989.67 & 17310.65 & 2505.74 & 398.99 & 1190.56 \\
\hline 17 Machinery appliances and equipment & 17047.31 & 183.92 & 21181.21 & 8018.29 & 520.44 & 591.57 & 21.35 & 366.06 & 4.46 & 659.15 & 410.74 & 343.31 & 18.91 & 6792.66 & 5693.89 & 840.83 & 227.79 & 235.07 \\
\hline 18 Miscellaneous manufacturing & 6246.93 & 129.76 & 6418.43 & 2658.04 & & 107.16 & 3.65 & 78.19 & 11 & 62.56 & 72.12 & & 5.04 & 1299.61 & 1751. & 271.55 & 220.86 & 89.08 \\
\hline 19 Electricity supply, gas and water & 17105.86 & 348.67 & 14251.70 & 7824.12 & & 674.33 & 103.20 & 1266.64 & 38.20 & 360.97 & & 645.33 & 81.52 & 5319.99 & 6729.85 & 1719.09 & 698.14 & 592.33 \\
\hline 20 Residential building construction & 26806.38 & 933.49 & 26995.50 & 15113. & 842.23 & 950.61 & 84.81 & 973.37 & 34.97 & 768.02 & 1128.04 & 1199.87 & 72.14 & 6580.25 & 10826.82 & 2107.40 & 1553.05 & 1001.77 \\
\hline 21 Other construction & 52314.32 & 1959.70 & 50071.7 & 30214 & 2122.66 & 2055.97 & & 2503.47 & 153.2 & & 2579.55 & 2293.26 & 279.13 & 13135.34 & 28729.94 & 3726.99 & 2685.47 & 1914.45 \\
\hline 22 Trade & 71984 & 2167.81 & 60058 & 3260 & 2457 & 2492. & 274. & 2114. & 105. & 2003 & 2432 & & 271.34 & 16583.38 & 23956.20 & 4867.40 & 2599.61 & 1736.02 \\
\hline nmodation, cafes and restaurants & & 832.33 & 15116 & 9780 & & 604. & 72.3 .3 & 657. & 43.5 & & & & 94.88 & 4420.59 & 6591.91 & 1500.31 & 1102.25 & 747.10 \\
\hline 24 Road transport & $\frac{13304.86}{506473}$ & 370.38 & 11898.46 & 6642. & 495.88 & 678.94 & 74.3 & 453. & 28.5 & 421. & 522. & 469.98 & 71.37 & 3351.94 & 5120.71 & 977.15 & 433.17 & 393.36 \\
\hline $\begin{array}{l}25 \text { Rail and pipeline transport } \\
26 \text { Other transport }\end{array}$ & $\frac{5064.73}{26587.56}$ & $\frac{8.21}{441.43}$ & $\frac{1749.17}{2081570}$ & $\begin{array}{r}2348.99 \\
1266075\end{array}$ & 113.20 & 107.61 & 27.36 & 625.55 & $\frac{15.02}{4287}$ & 393.62 & 328.93 & $\begin{array}{l}79.78 \\
15606\end{array}$ & 76.17 & 401.01 & 565.15 & $\begin{array}{r}55.35 \\
147777\end{array}$ & $\begin{array}{c}4.64 \\
01050\end{array}$ & 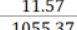 \\
\hline 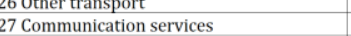 & 32223.41 & $\begin{array}{l}441.43 \\
466.03\end{array}$ & $\frac{20815.70}{22014.15}$ & $\frac{12660.75}{8756.32}$ & 3700.95 & $\begin{array}{l}49783 \\
348.38\end{array}$ & 22.30 & $\begin{array}{l}682.87 \\
274.19\end{array}$ & $\begin{array}{l}42.87 \\
19.95\end{array}$ & $\begin{array}{l}691.49 \\
191.53\end{array}$ & $\begin{array}{l}727.22 \\
573.40\end{array}$ & $\frac{1560.66}{386.98}$ & 35.84 & 46640.51 & $\begin{array}{r}8531.04 \\
4817.99\end{array}$ & 1411.777 & $\begin{array}{l}910.50 \\
1656.67\end{array}$ & $\begin{array}{l}105.37 \\
452.19\end{array}$ \\
\hline 28 Finance, property and business services & 145901.92 & 2397.65 & 111263.03 & 58187.66 & 2076.72 & 2447.26 & 160.08 & 2576.38 & 88.14 & 2052.57 & 2717.87 & 3015.66 & 253.29 & 24391.12 & 41252.66 & 5902.60 & 7983.44 & 2768.19 \\
\hline 29 Ownership of dwellings & 57211.92 & 1828.77 & 39079.29 & 30778.26 & 1573.48 & 1314.78 & 116.32 & 1207.34 & 27.37 & 1050.68 & 1616.49 & 1843.07 & 120.79 & 10109.70 & 19720.21 & 2959.68 & 2922.77 & 1531.36 \\
\hline ent administration and defence & 30346.18 & 731.46 & 21019.12 & 14902.17 & 889.89 & 1024.48 & 182.60 & 840.38 & 113.81 & 491.70 & 1921.54 & 1592.03 & 202.08 & 8375.08 & 10901.35 & 3022.32 & 10420.96 & 3323.26 \\
\hline 31 Education & 21235.51 & 758.41 & 18413.97 & 9926.29 & 765.35 & 869.05 & 77.32 & 706.20 & 39.60 & 405.17 & 837.65 & 832.21 & 95.23 & 5324.43 & 7854.84 & 1767.26 & 1571.99 & 785.55 \\
\hline 32 Health a & 30280.74 & 1161.61 & 24833.39 & 15172.65 & 1244.95 & 1087.85 & 112.93 & 830.92 & 47.92 & 531.46 & 1194.10 & 1213.87 & 116.82 & 8508.61 & 9669.51 & 2133.43 & 1542.99 & 939.80 \\
\hline 33 Cultur & 7824.36 & 231.43 & 7580.06 & 3964.88 & 119.04 & 143.84 & 9.57 & 107.41 & 12.84 & 74.26 & 213.25 & 366.63 & 13.40 & 1558.15 & 2474.99 & 623.62 & 563.02 & 426.21 \\
\hline 34 Personal and other services & 7417.86 & 192.32 & 6044.88 & 3549.17 & 211.30 & 217.90 & 19.94 & 184.24 & 9.49 & 141.83 & 244.10 & 323.05 & 29.42 & 1874.59 & 2616.43 & 512.73 & 489.27 & 328.01 \\
\hline
\end{tabular}


Table SI 2.2 Summary of Impact information.

\begin{tabular}{|c|c|c|c|c|}
\hline Aspect & Region & Sectors & Example impact & Reference \\
\hline Coal exports & All QLD & Coal, oil and gas & $\begin{array}{l}\text { "Queensland coal exports may have taken a } \$ 1.5 \text { billion hit from Cyclone } \\
\text { Debbie as more than } 22 \text { mines were forced to halt production while roads and } \\
\text { ports were shut." }\end{array}$ & $\begin{array}{l}\text { (John McCarthy } \\
\text { and Vogler, 2017) }\end{array}$ \\
\hline Coal exports & All QLD & Coal, oil and gas & $\begin{array}{l}\text { "Landslides caused by Debbie have halted operations on the busiest rail line } \\
\text { connecting coking coal mines in Queensland to ports, and rail operator } \\
\text { Aurizon said it would take about five weeks to repair. BHP Billiton, the world's } \\
\text { biggest shipper of coking coal, said it won't meet its export commitments from } \\
\text { its mines in Queensland's Bowen Basin - among five miners in the region to } \\
\text { declare force majeure, a clause typically invoked after natural disasters." }\end{array}$ & (Serapio, 2017) \\
\hline Coal exports & $\begin{array}{l}\text { Parts of QLD- } \\
\text { Fitzroy, QLD- } \\
\text { Mackay }\end{array}$ & Coal, oil and gas & $\begin{array}{l}\text { Mining operator Aurizon said it expected to lose up to } \$ 115 \text { million as a result } \\
\text { of Cyclone Debbie influencing mines at Blackwater, Moura, Goonyella, and } \\
\text { Newlands. }\end{array}$ & $\begin{array}{l}\text { (O'Brien, 2017) } \\
\text { (Public-Private } \\
\text { Partnership in } \\
\text { Infrastructure } \\
\text { Resource Centre, } \\
\text { 2016) }\end{array}$ \\
\hline $\begin{array}{l}\text { Business } \\
\text { Flooding }\end{array}$ & $\begin{array}{l}\text { NSW Richmond } \\
\text { \& Tweed }\end{array}$ & $\begin{array}{l}\text { Accommodation, } \\
\text { Cafes and } \\
\text { Restaurants, } \\
\text { Trade, }\end{array}$ & $\begin{array}{l}\text { "This is a community of at least } 50,000 \text { people and when you go for a drive, } \\
\text { come back and six months you'll see } 50 \text { to } 80 \text { per cent of these businesses not } \\
\text { open." }\end{array}$ & $\begin{array}{l}\text { (Gleeson, April 3, } \\
2017 \text { 1:36pm) }\end{array}$ \\
\hline $\begin{array}{l}\text { Business } \\
\text { Flooding }\end{array}$ & $\begin{array}{l}\text { NSW Richmond } \\
\text { \& Tweed }\end{array}$ & $\begin{array}{l}\text { Accommodation, } \\
\text { Cafes and } \\
\text { Restaurants, } \\
\text { Trade, }\end{array}$ & $\begin{array}{l}\text { "Half of the small businesses that make up the four streets of the Lismore } \\
\text { central business district may have to close up shop - for good." }\end{array}$ & $\begin{array}{l}\text { (Hansen, April 9, } \\
2017 \text { 12:00am) }\end{array}$ \\
\hline Tourism impacts & QLD - Mackay & Multiple & $\begin{array}{l}1.3 \text { million visitors spending some } \$ 967 \text { million (1). } \\
\text { Debbie has been confirmed as causing 'catastrophic damage and is expected to } \\
\text { be worse with businesses on Hamilton Island questioning whether rebuilding } \\
\text { is the best option. Cyclone Yasi cost tourism } 400 \text { million (2) indicating } \\
\$ 200 \text { million an approximation for Debbie. }\end{array}$ & $\begin{array}{l}\text { (1) } \\
\text { (Tourism } \\
\text { Research } \\
\text { Australia, 2016) } \\
(2) \\
\text { (Flaherty, 2017) }\end{array}$ \\
\hline Sugar Cane & QLD- Mackay & $\begin{array}{l}\text { Sugar cane } \\
\text { growing }\end{array}$ & $\begin{array}{l}\text { "The sugar cane industry has forecast a } \$ 150 \mathrm{~m} \text { blow, and the Bowen region's } \\
\$ 450 \mathrm{~m} \text {-a-year horticulture industry will also suffer heavy losses that could } \\
\text { cause the price of household staples such as tomatoes to rise later in the year." }\end{array}$ & $\begin{array}{l}\text { (Robertson, } \\
2017)\end{array}$ \\
\hline Sugar Cane & QLD- Mackay & $\begin{array}{l}\text { Sugar cane } \\
\text { growing }\end{array}$ & $\begin{array}{l}\text { "Damage to Queensland's sugar industry is expected to cost } \mathrm{A} \$ 150 \text { million } \\
\text { (US } \$ 114.4 \text { million). The majority of these costs lie in Proserpine and Mackay. } \\
35 \% \text { of all sugarcane in the Proserpine region and } 20 \% \text { of all sugarcane in the } \\
\text { Mackay region have been damaged, costing } A \$ 50 \text { million and A } \$ 81 \text { million }\end{array}$ & $\begin{array}{l}\text { (The Courier } \\
\text { Mail, 2017) } \\
\text { (QFF, 2017) }\end{array}$ \\
\hline
\end{tabular}




\begin{tabular}{|c|c|c|c|c|}
\hline & & & $\begin{array}{l}\text { respectively." Gross value of Sugar cane - Cut for crushing (SA4 Mackay } \\
\text { region): 354M (2014/15) ABS 7503.0 }\end{array}$ & \\
\hline Agriculture & QLD-Mackay & $\begin{array}{l}\text { Other } \\
\text { agriculture }\end{array}$ & $\begin{array}{l}\text { "Canegrowers around Proserpine and Mackay, and the horticulture industry at } \\
\text { Bowen - including tomato, capsicum and eggplant producers - have been } \\
\text { the hardest hit." }\end{array}$ & $\begin{array}{l}\text { (Donoughue, } \\
\text { 2017) }\end{array}$ \\
\hline Vegetables & QLD-Mackay & $\begin{array}{l}\text { Other } \\
\text { agriculture }\end{array}$ & $\begin{array}{l}\text { "The Queensland Farmers Federation (QFF) said early figures show actual } \\
\text { crop damage to Bowen's vegetable industry is about } \$ 100 \text { million, accounting } \\
\text { for about } 20 \text { per cent of the season's crop." }\end{array}$ & $\begin{array}{l}\text { (Donoughue, } \\
\text { 2017) }\end{array}$ \\
\hline Vegetables & $\begin{array}{l}\text { QLD-Mackay, } \\
\text { QLD Northern }\end{array}$ & $\begin{array}{l}\text { Other } \\
\text { agriculture }\end{array}$ & "Winter crops lost in Bowen-Gumlu region \$A100 million" & (Emerick, 2017) \\
\hline Vegetables & QLD-Mackay & $\begin{array}{l}\text { Other } \\
\text { agriculture }\end{array}$ & $\begin{array}{l}\text { "All vegetables in the ground in the Bowen and Whitsunday region have been } \\
\text { lost. Over } \$ 100 \text { million worth of produce has been written off." Nurseries, cut } \\
\text { flowers or cultivated turf, Fruit and nuts (excluding grapes), Vegetables for } \\
\text { human consumption - Total (SA4 Mackay region): 188M (2014/15) ABS } \\
7503.0\end{array}$ & (QFF, 2017) \\
\hline Vegetables & $\begin{array}{l}\text { NSW Richmond } \\
\text { \& Tweed }\end{array}$ & $\begin{array}{l}\text { Other } \\
\text { agriculture }\end{array}$ & $\begin{array}{l}\text { Australian Macadamia Society chief executive Jolyon Burnett said that lost nut } \\
\text { would have been worth about } \$ 8 \text { million. Soybeans - "To date, } \$ 3.75 \text { million in } \\
\text { crops have been reported lost in the Northern Rivers, and another } \$ 445,000 \text { of } \\
\text { beans downgraded." }\end{array}$ & (Honan, 2017) \\
\hline $\begin{array}{l}\text { Agriculture, } \\
\text { grains and } \\
\text { sugarcane }\end{array}$ & All 19 regions & $\begin{array}{l}\text { Grains, } \\
\text { Other } \\
\text { agriculture, } \\
\text { Sugar cane } \\
\text { growing }\end{array}$ & $\begin{array}{l}\text { STATE RECOVERY PLAN 2017-2019 Operation Queensland Recovery - "The } \\
\text { National Farmers' Federation has cited industry groups estimating damage to } \\
\text { crops of up to } \$ 1 \text { billion." }\end{array}$ & $\begin{array}{l}\text { (Queensland } \\
\text { Government, } \\
\text { 2017) }\end{array}$ \\
\hline Dairy & QLD - Brisbane & $\begin{array}{l}\text { Dairy cattle and } \\
\text { pigs }\end{array}$ & $\begin{array}{l}\text { "it is anticipated that the cost to the farming industry in South East } \\
\text { Queensland will be in excess of } \$ 6 \text { million." "So far major damages assessed } \\
\text { have included: more than } \$ 300,000 \text { in losses to equipment including pumps, } \\
\text { tractors, irrigation equipment; more than } 200 \text { hectares and } 400 \text { hectares of } \\
\text { pasture lost; } 121 \text { km of fencing destroyed; more than } 10 \text { km of internal } \\
\text { laneways damaged; more than } 400 \text { round bales hay lost; and the loss of } 11 \\
\text { animals." Queensland Dairyfarmers' Organisation (QDO) report } 50 \text { dairy } \\
\text { farms have been impacted South East QLD. Eight reported to have severe } \\
\text { flooding through Logan and the Gold coast with some reporting } 80 \% \text { plus } \\
\text { stock loss and severely damaged on farm infrastructure. Growcom have } \\
\text { surveyed } 12 \text { farmers to date in SEQ. Several vegetable growers are reporting } \\
\text { widespread flood damage, soil erosion, loss of irrigation equipment, some } \\
\text { infrastructure damage and crop losses. } \\
\text { http://www.dairypage.com.au/newsroom/2017/4/12/cyclone-debbies-6- } \\
\text { million-impact-on-dairy }\end{array}$ & (Neville, 2017) \\
\hline Urban & QLD - Central & Multiple sectors & $\begin{array}{l}\text { Central Queensland: Rockhampton has started to be inundated with slow } \\
\text { moving flood waters, the flood will peak of } 9.0 \text { meters today }(06 / 04 / 2017) \text {. } \\
\text { The slow-moving flood will effect a number of industries with Growcom and }\end{array}$ & (QFF, 2017) \\
\hline
\end{tabular}




\begin{tabular}{|c|c|c|c|c|}
\hline & & & $\begin{array}{l}\text { NGIQ watching the region closely. Cotton is likely to have some damage } \\
\text { through Central Queensland as well. With Cotton Australia ensuring that } \\
\text { damage is reported through to the Government, although with the sparsity of } \\
\text { the damage in these western regions they are investigating Individual Disaster } \\
\text { Declarations for effected farms. }\end{array}$ & \\
\hline Infrastructure & All QLD & Multiple sectors & $\begin{array}{l}\text { (1) "Queensland Premier Annastacia Palaszczuk said the cost of recovery } \\
\text { would 'be in the billions' of dollars, with roads, bridges, crops, homes and } \\
\text { schools all needing serious repairs." } \\
\text { (2) Cyclone Yasi was "the cost of rebuilding public infrastructure and } \\
\text { supporting businesses and the community following these disasters totals } \\
\text { approximately } \$ 6.8 \text { billion" (overlaps with other costs) and likely similar for } \\
\text { Debbie. }\end{array}$ & $\begin{array}{l}\text { (1) (Gillman, } \\
\text { 2017) } \\
\text { (2) (Australian } \\
\text { Government, } \\
\text { 2011) }\end{array}$ \\
\hline $\begin{array}{l}\text { Disaster } \\
\text { assistance }\end{array}$ & $\begin{array}{l}\text { QLD most } \\
\text { regions. }\end{array}$ & Multiple sectors & $\begin{array}{l}\text { Minister Ryan said counter-disaster operations assistance will be made } \\
\text { available to the eight local government areas of Burdekin, Charters Towers, } \\
\text { Isaac, Hinchinbrook, Mackay, Palm Island, Townsville and Whitsunday. }\end{array}$ & $\begin{array}{l}\text { (Carruthers, } \\
\text { 2017) }\end{array}$ \\
\hline Insurance & National & Multiple Sectors & $\begin{array}{l}\text { "Insurance losses \$A306 million”. Over a \$1bill in insurance claims but } \\
\text { probably includes a lot of business loss claims (eg.in Logan and Lismore). } \\
\text { https://www.cciq.com.au/news/cyclone-debbie-overall-damage-bill-and- } \\
\text { economic-impact }\end{array}$ & $\begin{array}{l}\text { (Underwriter, } \\
\text { 2017) }\end{array}$ \\
\hline Fatalities & - & - & $\begin{array}{l}12 \text { Fatalities. There are economic valuation estimates of value of life. Eg value } \\
\text { of a statistical life } \$ 4.2 \text { million. }\end{array}$ & $\begin{array}{l}\text { (Department of } \\
\text { the Prime } \\
\text { Minister and } \\
\text { Cabinet, 2014) }\end{array}$ \\
\hline Evacuation costs & - & - & 25,000 residents in Mackay, and 5,5000 Bowen evacuated. & $\begin{array}{l}\text { (BBC News, } \\
\text { 2017) (Killalea et } \\
\text { al., 2017) }\end{array}$ \\
\hline Schools & - & - & 400 schools closed. & $\begin{array}{l}\text { (Agence-France- } \\
\text { Presse, 2017) }\end{array}$ \\
\hline Airflights & - & - & $\begin{array}{l}\text { Flights cancelled Townsville etc from March } 27 \\
\text { Virgin Airlines loss in the } 3 \text { months to March } \$ 62.3 \text { million was impacted by } \\
\text { Cyclone Debbie }\end{array}$ & $\begin{array}{l}\text { (Courier Mail, } \\
\text { 2017) }\end{array}$ \\
\hline Rail & - & - & $\begin{array}{l}\text { QLD Rail suspended trains between Rocky and Townsville. North Queensland } \\
\text { Bulk Ports closed at Mackay, Abbot Point and Hay Point. }\end{array}$ & $\begin{array}{l}\text { (North } \\
\text { Queensland Bulk } \\
\text { Ports } \\
\text { Corporation, } \\
\text { 2017) }\end{array}$ \\
\hline $\begin{array}{l}\text { Emergency } \\
\text { workers }\end{array}$ & - & - & 1000 emergency workers deployed, 200 Energex workers. & (Cummins, 2017) \\
\hline Defence forces & - & - & ADF deployed 1200 personnel. & $\begin{array}{l}\text { (Young et al., } \\
2017)\end{array}$ \\
\hline
\end{tabular}


Table SI 2.3 Summary of major infrastructure damage and impacts by region

\begin{tabular}{|c|c|c|}
\hline Region & Local council region & Major infrastructure (building, roads and transport) damage and impacts \\
\hline QLD - Mackay & Whitsunday & $\begin{array}{l}\text { - Whitsunday Coast Airport closed for six days. } \\
\text { - Shute Harbour destroyed. } \\
\text { - Substantial roads and bridges damaged. } \\
\text { - Power disrupted for a number of days. }\end{array}$ \\
\hline QLD - Mackay & Mackay & $\begin{array}{l}\text { - Substantial roads damaged. } \\
\text { - Iconic community assets destroyed or damaged. } \\
\text { - Parks and places of cultural significance damaged. }\end{array}$ \\
\hline QLD - Mackay & Isaac & $\begin{array}{l}\text { - Substantial roads and bridges damaged. } \\
\text { - Moranbah Airport closed for a number of days. } \\
\text { - Power disrupted for a number of days. } \\
\text { - Telecommunications and internet network disrupted for a significant number of days. }\end{array}$ \\
\hline QLD - Fitzroy & Rockhampton & $\begin{array}{l}\text { - Rockhampton Airport closed for ten days. } \\
\text { - Rural roads damaged. }\end{array}$ \\
\hline QLD - Fitzroy & Livingstone Shire & - Roads damaged. \\
\hline QLD - Darling Downs & Scenic Rim & - Substantial roads and bridges damaged. \\
\hline QLD - Brisbane & City of Logan & $\begin{array}{l}\text { - Public transport network disrupted for five days. } \\
\text { - Road and bridges damaged. } \\
\text { - } 46 \text { sport and recreation facilities were impacted from weeks to months. } \\
\text { - Five wastewater pump stations damaged. } \\
\text { - Power disrupted for a number of days. }\end{array}$ \\
\hline QLD - Brisbane & City of Gold Coast & - Substantial roads and bridges damaged. \\
\hline
\end{tabular}




\section{SI 3: Raw data for production layer decomposition graphs}

Table SI 3.1 Production layer decomposition for the indicator Employment (values are in Full-time Equivalent (FTE)). Region acronyms: RemNSW: Rest of New South Wales (NSW); NSW-Rm\&T: NSW Richmond \& Tweed; VIC: Victoria; QLD-B: Queensland (QLD) - Brisbane; QLD-WBB: Wide Bay Burnett; QLD-DD: Darling Downs, QL-SW: South West; QLD-F: Fitzroy; QLD-CW: Central West; QLD-M: Mackay; QLD-N: Northern; QLD-FN: Far North; QLD-NW: North West; SA: South Australia; WA: Western Australia; TAS: Tasmania; ACT: Australian Capital Territory; NT: Northern Territory.

\begin{tabular}{|c|c|c|c|c|c|c|c|c|c|c|c|c|c|c|c|c|c|c|}
\hline $\begin{array}{c}\text { Production } \\
\text { Layer }\end{array}$ & RemNSW & NSW-Rm\&T & VIC & QLD-B & QLD-WBB & QLD-DD & QLD-SW & QLD-F & QLD-CW & QLD-M & QLD-N & QLD-FN & QLD-NW & SA & WA & TAS & ACT & NT \\
\hline 1.00 & 0.00 & -2.59 & 0.00 & 0.00 & 0.00 & 0.00 & 0.00 & -22.84 & -0.51 & -34.10 & -2.78 & 0.00 & 0.00 & 0.00 & 0.00 & 0.00 & 0.00 & 0.00 \\
\hline 2.00 & 0.00 & -1727.97 & 0.00 & -563.58 & 0.00 & 0.00 & 0.00 & -770.84 & -24.07 & -1424.52 & -228.04 & 0.00 & 0.00 & 0.00 & 0.00 & 0.00 & 0.00 & 0.00 \\
\hline 3.00 & -13.13 & -1728.48 & -10.48 & -570.12 & -0.45 & -0.46 & -0.06 & -772.01 & -24.11 & -1425.73 & -229.08 & -0.49 & -0.08 & -3.08 & -7.31 & -0.86 & -0.64 & -0.40 \\
\hline 4.00 & -418.69 & -1811.48 & -351.08 & -833.30 & -15.43 & -16.28 & -2.11 & -823.47 & -25.52 & -1507.73 & -268.42 & -16.42 & -1.95 & -105.51 & -188.74 & -29.57 & -20.32 & -11.83 \\
\hline 5.00 & -479.65 & -1812.50 & -397.92 & -859.59 & -16.80 & -17.73 & -2.26 & -824.94 & -25.58 & -1508.91 & -270.04 & -17.90 & -2.13 & -117.87 & -209.70 & -32.46 & -22.16 & -13.04 \\
\hline 6.00 & -713.02 & -1825.57 & -595.40 & -981.31 & -23.65 & -25.18 & -3.43 & -834.47 & -26.25 & -1520.68 & -279.83 & -25.14 & -3.28 & -171.60 & -292.81 & -46.18 & -31.23 & -18.17 \\
\hline 7.00 & -767.10 & $\begin{array}{l}-1826.48 \\
\end{array}$ & -636.91 & -1004.51 & -24.92 & -26.53 & -3.61 & -835.78 & -26.34 & -1521.75 & -281.30 & -26.53 & -3.59 & -182.73 & -311.65 & -48.81 & -32.77 & -19.25 \\
\hline 8.00 & -878.97 & -1829.16 & -729.93 & -1058.06 & -27.45 & -29.30 & -3.95 & -838.57 & -26.51 & -1524.31 & -284.34 & -29.37 & -4.02 & -206.40 & -349.26 & -54.35 & -36.77 & -21.37 \\
\hline 9.00 & -914.74 & -1829.76 & -757.61 & -1073.47 & -28.28 & -30.18 & -4.07 & -839.43 & -26.57 & -1525.01 & -285.30 & -30.30 & -4.23 & -213.81 & -361.66 & -56.09 & -37.81 & -22.08 \\
\hline 10.00 & -967.46 & -1830.69 & -801.04 & -1097.62 & -29.35 & -31.34 & -4.21 & -840.56 & -26.63 & -1525.94 & -286.55 & -31.53 & -4.43 & -224.39 & -378.96 & -58.52 & -39.63 & -23.03 \\
\hline 11.00 & $\begin{array}{l}-988.58 \\
\end{array}$ & $\begin{array}{l}-1831.04 \\
\end{array}$ & -817.52 & -1106.76 & -29.82 & -31.85 & -4.28 & -841.05 & -26.67 & -1526.34 & -287.10 & -32.07 & -4.55 & -228.74 & -386.19 & -59.54 & -40.26 & -23.44 \\
\hline 12.00 & -1013.54 & -1831.44 & -837.93 & -1117.95 & -30.31 & -32.37 & -4.34 & -841.57 & -26.70 & -1526.76 & -287.67 & -32.64 & -4.64 & -233.64 & -394.31 & -60.66 & -41.10 & -23.89 \\
\hline
\end{tabular}

Table SI 3.2 Production layer decomposition for the indicator Value-added (values are in AUD \$m). Region acronyms: RemNSW: Rest of New South Wales (NSW); NSW-Rm\&T: NSW Richmond \& Tweed; VIC: Victoria; QLD-B: Queensland (QLD) - Brisbane; QLD-WBB: Wide Bay Burnett; QLD-DD: Darling Downs, QL-SW: South West; QLD-F: Fitzroy; QLD-CW: Central West; QLD-M: Mackay; QLD-N: Northern; QLD-FN: Far North; QLD-NW: North West; SA: South Australia; WA: Western Australia; TAS: Tasmania; ACT: Australian Capital Territory; NT: Northern Territory.

\begin{tabular}{|c|c|c|c|c|c|c|c|c|c|c|c|c|c|c|c|c|c|c|}
\hline $\begin{array}{c}\text { Production } \\
\text { Layer }\end{array}$ & RemNSW & NSW-Rm\&T & VIC & QLD-B & QLD-WBB & QLD-DD & QLD-SW & QLD-F & QLD-CW & QLD-M & QLD-N & QLD-FN & QLD-NW & SA & WA & TAS & ACT & NT \\
\hline 1.00 & 0.00 & -0.51 & 0.00 & 0.00 & 0.00 & 0.00 & 0.00 & -28.23 & -0.10 & -39.78 & -3.42 & 0.00 & 0.00 & 0.00 & 0.00 & 0.00 & 0.00 & 0.00 \\
\hline 2.00 & 0.00 & -152.33 & 0.00 & -53.47 & 0.00 & 0.00 & 0.00 & -480.87 & -4.47 & -691.19 & -89.57 & 0.00 & 0.00 & 0.00 & 0.00 & 0.00 & 0.00 & 0.00 \\
\hline 3.00 & -2.68 & -152.43 & -1.90 & -54.85 & -0.12 & -0.12 & -0.02 & -481.34 & -4.48 & -691.69 & -89.83 & -0.10 & -0.02 & -0.62 & -2.45 & -0.17 & -0.11 & -0.09 \\
\hline 4.00 & -79.79 & -166.50 & -61.45 & -104.05 & -3.25 & -3.33 & -0.48 & -493.96 & -4.73 & -709.75 & -97.86 & -3.02 & -0.44 & -19.41 & -50.92 & -5.46 & -3.49 & -2.29 \\
\hline 5.00 & -94.32 & -166.71 & -69.49 & -110.49 & -3.63 & -3.72 & -0.53 & -494.39 & -4.75 & -710.10 & -98.30 & -3.34 & -0.48 & -22.09 & -56.73 & -6.02 & -3.75 & -2.56 \\
\hline 6.00 & -137.22 & -168.81 & -103.60 & -132.84 & -4.86 & -5.01 & -0.70 & -496.54 & -4.82 & -712.72 & -100.14 & -4.60 & -0.72 & -31.53 & -73.79 & -8.41 & -5.31 & -3.48 \\
\hline 7.00 & -148.81 & -168.99 & -110.72 & -137.95 & -5.16 & -5.32 & -0.73 & -496.91 & -4.84 & -713.05 & -100.50 & -4.89 & -0.80 & -33.78 & -78.68 & -8.91 & -5.55 & -3.72 \\
\hline 8.00 & -169.93 & -169.45 & -127.05 & -147.98 & -5.64 & -5.82 & -0.79 & -497.59 & -4.86 & -713.74 & -101.09 & -5.41 & -0.91 & -38.05 & -86.38 & -9.89 & -6.24 & -4.11 \\
\hline 9.00 & -177.26 & -169.56 & -131.83 & -151.21 & -5.83 & -6.01 & -0.82 & -497.83 & -4.87 & -713.96 & -101.32 & -5.60 & -0.97 & -39.51 & -89.45 & -10.22 & -6.40 & -4.26 \\
\hline 10.00 & -187.34 & -169.72 & -139.48 & -155.79 & -6.04 & -6.23 & -0.84 & -498.12 & -4.88 & -714.23 & -101.57 & -5.82 & -1.03 & -41.44 & -93.08 & -10.66 & -6.71 & -4.44 \\
\hline 11.00 & -191.59 & -169.78 & -142.35 & -157.66 & -6.14 & -6.34 & -0.85 & -498.26 & -4.89 & -714.36 & -101.70 & -5.93 & -1.06 & -42.28 & $\begin{array}{l}-94.81 \\
\end{array}$ & -10.85 & -6.82 & -4.53 \\
\hline 12.00 & -196.40 & -169.85 & -145.95 & -159.80 & -6.24 & -6.44 & -0.87 & -498.39 & -4.89 & -714.48 & -101.81 & -6.04 & -1.09 & -43.19 & -96.55 & -11.05 & -6.96 & -4.61 \\
\hline
\end{tabular}


SI 4: Underlying raw data for main text's Figure 3.

\begin{tabular}{|c|c|c|c|c|}
\hline Region & $\begin{array}{l}\text { Sector } \\
\text { number }\end{array}$ & Sector name & $\begin{array}{c}\text { Employment } \\
\text { Log10 (FTE) }\end{array}$ & $\begin{array}{c}\text { Value added } \\
\text { Log10(AUD\$m) }\end{array}$ \\
\hline Rest of NSW & 1 & Sheep & -0.902 & 0.000 \\
\hline Rest of NSW & 2 & Grains & -0.925 & -0.230 \\
\hline Rest of NSW & 3 & Beef cattle & -1.072 & 0.000 \\
\hline Rest of NSW & 4 & Dairy cattle and pigs & -0.329 & 0.000 \\
\hline Rest of NSW & 5 & Other agriculture & -1.230 & -0.525 \\
\hline Rest of NSW & 6 & Sugar cane growing & 0.000 & 0.000 \\
\hline Rest of NSW & 7 & Forestry and fishing & -0.290 & 0.000 \\
\hline Rest of NSW & 8 & Coal, oil and gas & -1.205 & -1.301 \\
\hline Rest of NSW & 9 & Non-ferrous metal ores & -0.469 & -0.071 \\
\hline Rest of NSW & 10 & Other mining & -0.718 & -0.447 \\
\hline Rest of NSW & 11 & Food manufacturing & -1.506 & -0.706 \\
\hline Rest of NSW & 12 & Textiles, clothing and footwear & -0.600 & 0.000 \\
\hline Rest of NSW & 13 & Wood and paper manufacturing & -1.004 & -0.204 \\
\hline Rest of NSW & 14 & Chemicals, petroleum and coal products & -1.348 & -0.752 \\
\hline Rest of NSW & 15 & Non-metallic mineral products & -0.816 & -0.080 \\
\hline Rest of NSW & 16 & Metals, metal products & -1.386 & -0.748 \\
\hline Rest of NSW & 17 & Machinery appliances and equipment & -1.263 & -0.365 \\
\hline Rest of NSW & 18 & Miscellaneous manufacturing & -1.030 & -0.139 \\
\hline Rest of NSW & 19 & Electricity supply, gas and water & -1.367 & -0.842 \\
\hline Rest of NSW & 20 & Residential building construction & -0.766 & 0.000 \\
\hline Rest of NSW & 21 & Other construction & -1.689 & -0.848 \\
\hline Rest of NSW & 22 & Trade & -2.297 & -1.328 \\
\hline Rest of NSW & 23 & Accommodation, cafes and restaurants & -1.519 & -0.375 \\
\hline Rest of NSW & 24 & Road transport & -1.649 & -0.665 \\
\hline Rest of NSW & 25 & Rail and pipeline transport & -1.382 & -0.655 \\
\hline Rest of NSW & 26 & Other transport & -1.712 & -1.068 \\
\hline Rest of NSW & 27 & Communication services & -1.525 & -1.001 \\
\hline Rest of NSW & 28 & Finance, property and business services & -2.507 & -1.793 \\
\hline Rest of NSW & 29 & Ownership of dwellings & -1.063 & -1.135 \\
\hline Rest of NSW & 30 & Government administration and defence & -1.269 & -0.287 \\
\hline Rest of NSW & 31 & Education & -1.327 & -0.284 \\
\hline Rest of NSW & 32 & Health and community services & -0.543 & 0.000 \\
\hline Rest of NSW & 33 & Cultural and recreational services & -0.724 & 0.000 \\
\hline Rest of NSW & 34 & Personal and other services & -1.199 & -0.079 \\
\hline NSW-Rm\&T & 35 & Sheep & 0.000 & 0.000 \\
\hline
\end{tabular}




\begin{tabular}{|c|c|c|c|c|}
\hline NSW-Rm\&T & 36 & Grains & 0.000 & 0.000 \\
\hline NSW-Rm\&T & 37 & Beef cattle & -0.493 & 0.000 \\
\hline NSW-Rm\&T & 38 & Dairy cattle and pigs & 0.000 & 0.000 \\
\hline NSW-Rm\&T & 39 & Other agriculture & -1.975 & -1.270 \\
\hline NSW-Rm\&T & 40 & Sugar cane growing & 0.000 & 0.000 \\
\hline NSW-Rm\&T & 41 & Forestry and fishing & 0.000 & 0.000 \\
\hline NSW-Rm\&T & 42 & Coal, oil and gas & 0.000 & 0.000 \\
\hline NSW-Rm\&T & 43 & Non-ferrous metal ores & 0.000 & 0.000 \\
\hline NSW-Rm\&T & 44 & Other mining & 0.000 & 0.000 \\
\hline NSW-Rm\&T & 45 & Food manufacturing & -1.261 & -0.461 \\
\hline NSW-Rm\&T & 46 & Textiles, clothing and footwear & -0.029 & 0.000 \\
\hline NSW-Rm\&T & 47 & Wood and paper manufacturing & -0.241 & 0.000 \\
\hline NSW-Rm\&T & 48 & Chemicals, petroleum and coal products & -0.004 & 0.000 \\
\hline NSW-Rm\&T & 49 & Non-metallic mineral products & 0.000 & 0.000 \\
\hline NSW-Rm\&T & 50 & Metals, metal products & 0.000 & 0.000 \\
\hline NSW-Rm\&T & 51 & Machinery appliances and equipment & -0.217 & 0.000 \\
\hline NSW-Rm\&T & 52 & Miscellaneous manufacturing & -0.199 & 0.000 \\
\hline NSW-Rm\&T & 53 & Electricity supply, gas and water & -0.321 & 0.000 \\
\hline NSW-Rm\&T & 54 & Residential building construction & -1.362 & -0.402 \\
\hline NSW-Rm\&T & 55 & Other construction & -1.320 & -0.479 \\
\hline NSW-Rm\&T & 56 & Trade & -2.592 & -1.622 \\
\hline NSW-Rm\&T & 57 & Accommodation, cafes and restaurants & -3.055 & -1.911 \\
\hline NSW-Rm\&T & 58 & Road transport & -1.387 & -0.402 \\
\hline NSW-Rm\&T & 59 & Rail and pipeline transport & 0.000 & 0.000 \\
\hline NSW-Rm\&T & 60 & Other transport & -0.445 & 0.000 \\
\hline NSW-Rm\&T & 61 & Communication services & -0.425 & 0.000 \\
\hline NSW-Rm\&T & 62 & Finance, property and business services & -1.235 & -0.522 \\
\hline NSW-Rm\&T & 63 & Ownership of dwellings & -0.146 & -0.218 \\
\hline NSW-Rm\&T & 64 & Government administration and defence & -0.160 & 0.000 \\
\hline NSW-Rm\&T & 65 & Education & -0.163 & 0.000 \\
\hline NSW-Rm\&T & 66 & Health and community services & -1.762 & -0.684 \\
\hline NSW-Rm\&T & 67 & Cultural and recreational services & -1.351 & -0.359 \\
\hline NSW-Rm\&T & 68 & Personal and other services & -0.204 & 0.000 \\
\hline VIC & 69 & Sheep & -0.725 & 0.000 \\
\hline VIC & 70 & Grains & -0.712 & -0.018 \\
\hline VIC & 71 & Beef cattle & -0.852 & 0.000 \\
\hline VIC & 72 & Dairy cattle and pigs & -0.898 & -0.083 \\
\hline VIC & 73 & Other agriculture & -1.217 & -0.512 \\
\hline VIC & 74 & Sugar cane growing & 0.000 & 0.000 \\
\hline VIC & 75 & Forestry and fishing & -0.108 & 0.000 \\
\hline VIC & 76 & Coal, oil and gas & -0.254 & -0.350 \\
\hline VIC & 77 & Non-ferrous metal ores & -0.164 & 0.000 \\
\hline VIC & 78 & Other mining & -0.513 & -0.242 \\
\hline
\end{tabular}




\begin{tabular}{|c|c|c|c|c|}
\hline VIC & 79 & Food manufacturing & -1.578 & -0.778 \\
\hline VIC & 80 & Textiles, clothing and footwear & -0.698 & 0.000 \\
\hline VIC & 81 & Wood and paper manufacturing & -1.044 & -0.244 \\
\hline VIC & 82 & Chemicals, petroleum and coal products & -1.397 & -0.801 \\
\hline VIC & 83 & Non-metallic mineral products & -0.721 & 0.000 \\
\hline VIC & 84 & Metals, metal products & -1.315 & -0.677 \\
\hline VIC & 85 & Machinery appliances and equipment & -1.392 & -0.494 \\
\hline VIC & 86 & Miscellaneous manufacturing & -1.075 & -0.184 \\
\hline VIC & 87 & Electricity supply, gas and water & -1.300 & -0.775 \\
\hline VIC & 88 & Residential building construction & -0.778 & 0.000 \\
\hline VIC & 89 & Other construction & -1.685 & -0.843 \\
\hline VIC & 90 & Trade & -2.236 & -1.266 \\
\hline VIC & 91 & Accommodation, cafes and restaurants & -1.389 & -0.245 \\
\hline VIC & 92 & Road transport & -1.619 & -0.634 \\
\hline VIC & 93 & Rail and pipeline transport & -0.821 & -0.094 \\
\hline VIC & 94 & Other transport & -1.603 & -0.958 \\
\hline VIC & 95 & Communication services & -1.349 & -0.825 \\
\hline VIC & 96 & Finance, property and business services & -2.385 & -1.672 \\
\hline VIC & 97 & Ownership of dwellings & -0.883 & -0.956 \\
\hline VIC & 98 & Government administration and defence & -1.106 & -0.124 \\
\hline VIC & 99 & Education & -1.277 & -0.234 \\
\hline VIC & 100 & Health and community services & -0.459 & 0.000 \\
\hline VIC & 101 & Cultural and recreational services & -0.763 & 0.000 \\
\hline VIC & 102 & Personal and other services & -1.127 & -0.007 \\
\hline QLD-B & 103 & Sheep & 0.000 & 0.000 \\
\hline QLD-B & 104 & Grains & 0.000 & 0.000 \\
\hline QLD-B & 105 & Beef cattle & -0.202 & 0.000 \\
\hline QLD-B & 106 & Dairy cattle and pigs & -1.752 & -0.937 \\
\hline QLD-B & 107 & Other agriculture & -1.045 & -0.340 \\
\hline QLD-B & 108 & Sugar cane growing & 0.000 & 0.000 \\
\hline QLD-B & 109 & Forestry and fishing & 0.000 & 0.000 \\
\hline QLD-B & 110 & Coal, oil and gas & -0.903 & -0.999 \\
\hline QLD-B & 111 & Non-ferrous metal ores & -0.090 & 0.000 \\
\hline QLD-B & 112 & Other mining & -0.705 & -0.434 \\
\hline QLD-B & 113 & Food manufacturing & -1.408 & -0.608 \\
\hline QLD-B & 114 & Textiles, clothing and footwear & -0.339 & 0.000 \\
\hline QLD-B & 115 & Wood and paper manufacturing & -0.736 & 0.000 \\
\hline QLD-B & 116 & Chemicals, petroleum and coal products & -1.094 & -0.498 \\
\hline QLD-B & 117 & Non-metallic mineral products & -0.599 & 0.000 \\
\hline QLD-B & 118 & Metals, metal products & -1.170 & -0.532 \\
\hline QLD-B & 119 & Machinery appliances and equipment & -1.078 & -0.180 \\
\hline QLD-B & 120 & Miscellaneous manufacturing & -0.793 & 0.000 \\
\hline QLD-B & 121 & Electricity supply, gas and water & -1.124 & -0.599 \\
\hline
\end{tabular}




\begin{tabular}{|c|c|c|c|c|}
\hline QLD-B & 122 & Residential building construction & -0.612 & 0.000 \\
\hline QLD-B & 123 & Other construction & -1.574 & -0.733 \\
\hline QLD-B & 124 & Trade & -2.483 & -1.514 \\
\hline QLD-B & 125 & Accommodation, cafes and restaurants & -2.463 & -1.319 \\
\hline QLD-B & 126 & Road transport & -1.751 & -0.767 \\
\hline QLD-B & 127 & Rail and pipeline transport & -1.039 & -0.312 \\
\hline QLD-B & 128 & Other transport & -1.441 & -0.796 \\
\hline QLD-B & 129 & Communication services & -1.047 & -0.523 \\
\hline QLD-B & 130 & Finance, property and business services & -2.185 & -1.471 \\
\hline QLD-B & 131 & Ownership of dwellings & -0.885 & -0.958 \\
\hline QLD-B & 132 & Government administration and defence & -1.080 & -0.098 \\
\hline QLD-B & 133 & Education & -1.063 & -0.020 \\
\hline QLD-B & 134 & Health and community services & -0.311 & 0.000 \\
\hline QLD-B & 135 & Cultural and recreational services & -1.291 & -0.298 \\
\hline QLD-B & 136 & Personal and other services & -1.023 & 0.000 \\
\hline QLD-WBB & 137 & Sheep & 0.000 & 0.000 \\
\hline QLD-WBB & 138 & Grains & 0.000 & 0.000 \\
\hline QLD-WBB & 139 & Beef cattle & -0.109 & 0.000 \\
\hline QLD-WBB & 140 & Dairy cattle and pigs & 0.000 & 0.000 \\
\hline QLD-WBB & 141 & Other agriculture & -0.549 & 0.000 \\
\hline QLD-WBB & 142 & Sugar cane growing & 0.000 & 0.000 \\
\hline QLD-WBB & 143 & Forestry and fishing & 0.000 & 0.000 \\
\hline QLD-WBB & 144 & Coal, oil and gas & 0.000 & -0.006 \\
\hline QLD-WBB & 145 & Non-ferrous metal ores & 0.000 & 0.000 \\
\hline QLD-WBB & 146 & Other mining & 0.000 & 0.000 \\
\hline QLD-WBB & 147 & Food manufacturing & -0.240 & 0.000 \\
\hline QLD-WBB & 148 & Textiles, clothing and footwear & 0.000 & 0.000 \\
\hline QLD-WBB & 149 & Wood and paper manufacturing & 0.000 & 0.000 \\
\hline QLD-WBB & 150 & Chemicals, petroleum and coal products & 0.000 & 0.000 \\
\hline QLD-WBB & 151 & Non-metallic mineral products & 0.000 & 0.000 \\
\hline QLD-WBB & 152 & Metals, metal products & 0.000 & 0.000 \\
\hline QLD-WBB & 153 & Machinery appliances and equipment & 0.000 & 0.000 \\
\hline QLD-WBB & 154 & Miscellaneous manufacturing & 0.000 & 0.000 \\
\hline QLD-WBB & 155 & Electricity supply, gas and water & -0.063 & 0.000 \\
\hline QLD-WBB & 156 & Residential building construction & 0.000 & 0.000 \\
\hline QLD-WBB & 157 & Other construction & -0.292 & 0.000 \\
\hline QLD-WBB & 158 & Trade & -0.797 & 0.000 \\
\hline QLD-WBB & 159 & Accommodation, cafes and restaurants & 0.000 & 0.000 \\
\hline QLD-WBB & 160 & Road transport & -0.161 & 0.000 \\
\hline QLD-WBB & 161 & Rail and pipeline transport & 0.000 & 0.000 \\
\hline QLD-WBB & 162 & Other transport & 0.000 & 0.000 \\
\hline QLD-WBB & 163 & Communication services & 0.000 & 0.000 \\
\hline QLD-WBB & 164 & Finance, property and business services & -0.545 & 0.000 \\
\hline
\end{tabular}




\begin{tabular}{|c|c|c|c|c|}
\hline QLD-WBB & 165 & Ownership of dwellings & 0.000 & 0.000 \\
\hline QLD-WBB & 166 & Government administration and defence & 0.000 & 0.000 \\
\hline QLD-WBB & 167 & Education & 0.000 & 0.000 \\
\hline QLD-WBB & 168 & Health and community services & 0.000 & 0.000 \\
\hline QLD-WBB & 169 & Cultural and recreational services & 0.000 & 0.000 \\
\hline QLD-WBB & 170 & Personal and other services & 0.000 & 0.000 \\
\hline QLD-DD & 171 & Sheep & 0.000 & 0.000 \\
\hline QLD-DD & 172 & Grains & -0.164 & 0.000 \\
\hline QLD-DD & 173 & Beef cattle & -0.243 & 0.000 \\
\hline QLD-DD & 174 & Dairy cattle and pigs & 0.000 & 0.000 \\
\hline QLD-DD & 175 & Other agriculture & -0.477 & 0.000 \\
\hline QLD-DD & 176 & Sugar cane growing & 0.000 & 0.000 \\
\hline QLD-DD & 177 & Forestry and fishing & 0.000 & 0.000 \\
\hline QLD-DD & 178 & Coal, oil and gas & 0.000 & -0.008 \\
\hline QLD-DD & 179 & Non-ferrous metal ores & 0.000 & 0.000 \\
\hline QLD-DD & 180 & Other mining & -0.029 & 0.000 \\
\hline QLD-DD & 181 & Food manufacturing & -0.299 & 0.000 \\
\hline QLD-DD & 182 & Textiles, clothing and footwear & 0.000 & 0.000 \\
\hline QLD-DD & 183 & Wood and paper manufacturing & 0.000 & 0.000 \\
\hline QLD-DD & 184 & Chemicals, petroleum and coal products & 0.000 & 0.000 \\
\hline QLD-DD & 185 & Non-metallic mineral products & 0.000 & 0.000 \\
\hline QLD-DD & 186 & Metals, metal products & 0.000 & 0.000 \\
\hline QLD-DD & 187 & Machinery appliances and equipment & 0.000 & 0.000 \\
\hline QLD-DD & 188 & Miscellaneous manufacturing & 0.000 & 0.000 \\
\hline QLD-DD & 189 & Electricity supply, gas and water & 0.000 & 0.000 \\
\hline QLD-DD & 190 & Residential building construction & 0.000 & 0.000 \\
\hline QLD-DD & 191 & Other construction & -0.279 & 0.000 \\
\hline QLD-DD & 192 & Trade & -0.806 & 0.000 \\
\hline QLD-DD & 193 & Accommodation, cafes and restaurants & 0.000 & 0.000 \\
\hline QLD-DD & 194 & Road transport & -0.281 & 0.000 \\
\hline QLD-DD & 195 & Rail and pipeline transport & 0.000 & 0.000 \\
\hline QLD-DD & 196 & Other transport & 0.000 & 0.000 \\
\hline QLD-DD & 197 & Communication services & 0.000 & 0.000 \\
\hline QLD-DD & 198 & Finance, property and business services & -0.629 & 0.000 \\
\hline QLD-DD & 199 & Ownership of dwellings & 0.000 & 0.000 \\
\hline QLD-DD & 200 & Government administration and defence & 0.000 & 0.000 \\
\hline QLD-DD & 201 & Education & 0.000 & 0.000 \\
\hline QLD-DD & 202 & Health and community services & 0.000 & 0.000 \\
\hline QLD-DD & 203 & Cultural and recreational services & 0.000 & 0.000 \\
\hline QLD-DD & 204 & Personal and other services & 0.000 & 0.000 \\
\hline QLD-SW & 205 & Sheep & 0.000 & 0.000 \\
\hline QLD-SW & 206 & Grains & 0.000 & 0.000 \\
\hline QLD-SW & 207 & Beef cattle & 0.000 & 0.000 \\
\hline
\end{tabular}




\begin{tabular}{|c|c|c|c|c|}
\hline QLD-SW & 208 & Dairy cattle and pigs & 0.000 & 0.000 \\
\hline QLD-SW & 209 & Other agriculture & 0.000 & 0.000 \\
\hline QLD-SW & 210 & Sugar cane growing & 0.000 & 0.000 \\
\hline QLD-SW & 211 & Forestry and fishing & 0.000 & 0.000 \\
\hline QLD-SW & 212 & Coal, oil and gas & 0.000 & 0.000 \\
\hline QLD-SW & 213 & Non-ferrous metal ores & 0.000 & 0.000 \\
\hline QLD-SW & 214 & Other mining & 0.000 & 0.000 \\
\hline QLD-SW & 215 & Food manufacturing & 0.000 & 0.000 \\
\hline QLD-SW & 216 & Textiles, clothing and footwear & 0.000 & 0.000 \\
\hline QLD-SW & 217 & Wood and paper manufacturing & 0.000 & 0.000 \\
\hline QLD-SW & 218 & Chemicals, petroleum and coal products & 0.000 & 0.000 \\
\hline QLD-SW & 219 & Non-metallic mineral products & 0.000 & 0.000 \\
\hline QLD-SW & 220 & Metals, metal products & 0.000 & 0.000 \\
\hline QLD-SW & 221 & Machinery appliances and equipment & 0.000 & 0.000 \\
\hline QLD-SW & 222 & Miscellaneous manufacturing & 0.000 & 0.000 \\
\hline QLD-SW & 223 & Electricity supply, gas and water & 0.000 & 0.000 \\
\hline QLD-SW & 224 & Residential building construction & 0.000 & 0.000 \\
\hline QLD-SW & 225 & Other construction & 0.000 & 0.000 \\
\hline QLD-SW & 226 & Trade & 0.000 & 0.000 \\
\hline QLD-SW & 227 & Accommodation, cafes and restaurants & 0.000 & 0.000 \\
\hline QLD-SW & 228 & Road transport & 0.000 & 0.000 \\
\hline QLD-SW & 229 & Rail and pipeline transport & 0.000 & 0.000 \\
\hline QLD-SW & 230 & Other transport & 0.000 & 0.000 \\
\hline QLD-SW & 231 & Communication services & 0.000 & 0.000 \\
\hline QLD-SW & 232 & Finance, property and business services & 0.000 & 0.000 \\
\hline QLD-SW & 233 & Ownership of dwellings & 0.000 & 0.000 \\
\hline QLD-SW & 234 & Government administration and defence & 0.000 & 0.000 \\
\hline QLD-SW & 235 & Education & 0.000 & 0.000 \\
\hline QLD-SW & 236 & Health and community services & 0.000 & 0.000 \\
\hline QLD-SW & 237 & Cultural and recreational services & 0.000 & 0.000 \\
\hline QLD-SW & 238 & Personal and other services & 0.000 & 0.000 \\
\hline QLD-F & 239 & Sheep & 0.000 & 0.000 \\
\hline QLD-F & 240 & Grains & 0.000 & 0.000 \\
\hline QLD-F & 241 & Beef cattle & -0.434 & 0.000 \\
\hline QLD-F & 242 & Dairy cattle and pigs & 0.000 & 0.000 \\
\hline QLD-F & 243 & Other agriculture & -0.074 & 0.000 \\
\hline QLD-F & 244 & Sugar cane growing & 0.000 & 0.000 \\
\hline QLD-F & 245 & Forestry and fishing & 0.000 & 0.000 \\
\hline QLD-F & 246 & Coal, oil and gas & -2.548 & -2.644 \\
\hline QLD-F & 247 & Non-ferrous metal ores & 0.000 & 0.000 \\
\hline QLD-F & 248 & Other mining & -0.333 & -0.062 \\
\hline QLD-F & 249 & Food manufacturing & -0.387 & 0.000 \\
\hline QLD-F & 250 & Textiles, clothing and footwear & 0.000 & 0.000 \\
\hline
\end{tabular}




\begin{tabular}{|c|c|c|c|c|}
\hline QLD-F & 251 & Wood and paper manufacturing & 0.000 & 0.000 \\
\hline QLD-F & 252 & Chemicals, petroleum and coal products & -0.127 & 0.000 \\
\hline QLD-F & 253 & Non-metallic mineral products & -0.078 & 0.000 \\
\hline QLD-F & 254 & Metals, metal products & -0.575 & 0.000 \\
\hline QLD-F & 255 & Machinery appliances and equipment & -0.262 & 0.000 \\
\hline QLD-F & 256 & Miscellaneous manufacturing & 0.000 & 0.000 \\
\hline QLD-F & 257 & Electricity supply, gas and water & -0.706 & -0.181 \\
\hline QLD-F & 258 & Residential building construction & -1.356 & -0.396 \\
\hline QLD-F & 259 & Other construction & -1.313 & -0.471 \\
\hline QLD-F & 260 & Trade & -2.204 & -1.235 \\
\hline QLD-F & 261 & Accommodation, cafes and restaurants & -1.283 & -0.138 \\
\hline QLD-F & 262 & Road transport & -1.983 & -0.998 \\
\hline QLD-F & 263 & Rail and pipeline transport & -1.345 & -0.618 \\
\hline QLD-F & 264 & Other transport & -0.933 & -0.288 \\
\hline QLD-F & 265 & Communication services & -0.715 & -0.192 \\
\hline QLD-F & 266 & Finance, property and business services & -1.018 & -0.305 \\
\hline QLD-F & 267 & Ownership of dwellings & 0.000 & 0.000 \\
\hline QLD-F & 268 & Government administration and defence & -0.223 & 0.000 \\
\hline QLD-F & 269 & Education & -0.037 & 0.000 \\
\hline QLD-F & 270 & Health and community services & 0.000 & 0.000 \\
\hline QLD-F & 271 & Cultural and recreational services & -1.360 & -0.368 \\
\hline QLD-F & 272 & Personal and other services & -1.870 & -0.750 \\
\hline QLD-CW & 273 & Sheep & 0.000 & 0.000 \\
\hline QLD-CW & 274 & Grains & 0.000 & 0.000 \\
\hline QLD-CW & 275 & Beef cattle & -0.428 & 0.000 \\
\hline QLD-CW & 276 & Dairy cattle and pigs & 0.000 & 0.000 \\
\hline QLD-CW & 277 & Other agriculture & -1.346 & -0.641 \\
\hline QLD-CW & 278 & Sugar cane growing & 0.000 & 0.000 \\
\hline QLD-CW & 279 & Forestry and fishing & 0.000 & 0.000 \\
\hline QLD-CW & 280 & Coal, oil and gas & 0.000 & 0.000 \\
\hline QLD-CW & 281 & Non-ferrous metal ores & 0.000 & 0.000 \\
\hline QLD-CW & 282 & Other mining & 0.000 & 0.000 \\
\hline QLD-CW & 283 & Food manufacturing & 0.000 & 0.000 \\
\hline QLD-CW & 284 & Textiles, clothing and footwear & 0.000 & 0.000 \\
\hline QLD-CW & 285 & Wood and paper manufacturing & 0.000 & 0.000 \\
\hline QLD-CW & 286 & Chemicals, petroleum and coal products & 0.000 & 0.000 \\
\hline QLD-CW & 287 & Non-metallic mineral products & 0.000 & 0.000 \\
\hline QLD-CW & 288 & Metals, metal products & 0.000 & 0.000 \\
\hline QLD-CW & 289 & Machinery appliances and equipment & 0.000 & 0.000 \\
\hline QLD-CW & 290 & Miscellaneous manufacturing & 0.000 & 0.000 \\
\hline QLD-CW & 291 & Electricity supply, gas and water & 0.000 & 0.000 \\
\hline QLD-CW & 292 & Residential building construction & 0.000 & 0.000 \\
\hline QLD-CW & 293 & Other construction & 0.000 & 0.000 \\
\hline
\end{tabular}




\begin{tabular}{|c|c|c|c|c|}
\hline QLD-CW & 294 & Trade & 0.000 & 0.000 \\
\hline QLD-CW & 295 & Accommodation, cafes and restaurants & 0.000 & 0.000 \\
\hline QLD-CW & 296 & Road transport & 0.000 & 0.000 \\
\hline QLD-CW & 297 & Rail and pipeline transport & 0.000 & 0.000 \\
\hline QLD-CW & 298 & Other transport & 0.000 & 0.000 \\
\hline QLD-CW & 299 & Communication services & 0.000 & 0.000 \\
\hline QLD-CW & 300 & Finance, property and business services & 0.000 & 0.000 \\
\hline QLD-CW & 301 & Ownership of dwellings & 0.000 & 0.000 \\
\hline QLD-CW & 302 & Government administration and defence & 0.000 & 0.000 \\
\hline QLD-CW & 303 & Education & 0.000 & 0.000 \\
\hline QLD-CW & 304 & Health and community services & 0.000 & 0.000 \\
\hline QLD-CW & 305 & Cultural and recreational services & 0.000 & 0.000 \\
\hline QLD-CW & 306 & Personal and other services & 0.000 & 0.000 \\
\hline QLD-M & 307 & Sheep & 0.000 & 0.000 \\
\hline QLD-M & 308 & Grains & -0.062 & 0.000 \\
\hline QLD-M & 309 & Beef cattle & -0.776 & 0.000 \\
\hline QLD-M & 310 & Dairy cattle and pigs & 0.000 & 0.000 \\
\hline QLD-M & 311 & Other agriculture & -2.337 & -1.632 \\
\hline QLD-M & 312 & Sugar cane growing & -0.986 & -0.170 \\
\hline QLD-M & 313 & Forestry and fishing & 0.000 & 0.000 \\
\hline QLD-M & 314 & Coal, oil and gas & -2.674 & -2.770 \\
\hline QLD-M & 315 & Non-ferrous metal ores & 0.000 & 0.000 \\
\hline QLD-M & 316 & Other mining & -0.379 & -0.108 \\
\hline QLD-M & 317 & Food manufacturing & -0.911 & -0.111 \\
\hline QLD-M & 318 & Textiles, clothing and footwear & 0.000 & 0.000 \\
\hline QLD-M & 319 & Wood and paper manufacturing & 0.000 & 0.000 \\
\hline QLD-M & 320 & Chemicals, petroleum and coal products & -0.194 & 0.000 \\
\hline QLD-M & 321 & Non-metallic mineral products & 0.000 & 0.000 \\
\hline QLD-M & 322 & Metals, metal products & -0.132 & 0.000 \\
\hline QLD-M & 323 & Machinery appliances and equipment & -0.478 & 0.000 \\
\hline QLD-M & 324 & Miscellaneous manufacturing & 0.000 & 0.000 \\
\hline QLD-M & 325 & Electricity supply, gas and water & -0.991 & -0.466 \\
\hline QLD-M & 326 & Residential building construction & -1.361 & -0.401 \\
\hline QLD-M & 327 & Other construction & -1.346 & -0.504 \\
\hline QLD-M & 328 & Trade & -2.078 & -1.108 \\
\hline QLD-M & 329 & Accommodation, cafes and restaurants & -2.627 & -1.483 \\
\hline QLD-M & 330 & Road transport & -2.157 & -1.173 \\
\hline QLD-M & 331 & Rail and pipeline transport & -0.338 & 0.000 \\
\hline QLD-M & 332 & Other transport & -0.550 & 0.000 \\
\hline QLD-M & 333 & Communication services & -1.011 & -0.488 \\
\hline QLD-M & 334 & Finance, property and business services & -1.094 & -0.381 \\
\hline QLD-M & 335 & Ownership of dwellings & 0.000 & 0.000 \\
\hline QLD-M & 336 & Government administration and defence & -0.074 & 0.000 \\
\hline
\end{tabular}




\begin{tabular}{|c|c|c|c|c|}
\hline QLD-M & 337 & Education & 0.000 & 0.000 \\
\hline QLD-M & 338 & Health and community services & 0.000 & 0.000 \\
\hline QLD-M & 339 & Cultural and recreational services & -1.450 & -0.457 \\
\hline QLD-M & 340 & Personal and other services & -0.289 & 0.000 \\
\hline QLD-N & 341 & Sheep & 0.000 & 0.000 \\
\hline QLD-N & 342 & Grains & 0.000 & 0.000 \\
\hline QLD-N & 343 & Beef cattle & 0.000 & 0.000 \\
\hline QLD-N & 344 & Dairy cattle and pigs & 0.000 & 0.000 \\
\hline QLD-N & 345 & Other agriculture & -0.225 & 0.000 \\
\hline QLD-N & 346 & Sugar cane growing & -0.737 & 0.000 \\
\hline QLD-N & 347 & Forestry and fishing & 0.000 & 0.000 \\
\hline QLD-N & 348 & Coal, oil and gas & -1.764 & -1.860 \\
\hline QLD-N & 349 & Non-ferrous metal ores & 0.000 & 0.000 \\
\hline QLD-N & 350 & Other mining & -0.418 & -0.147 \\
\hline QLD-N & 351 & Food manufacturing & -0.395 & 0.000 \\
\hline QLD-N & 352 & Textiles, clothing and footwear & 0.000 & 0.000 \\
\hline QLD-N & 353 & Wood and paper manufacturing & 0.000 & 0.000 \\
\hline QLD-N & 354 & Chemicals, petroleum and coal products & -0.111 & 0.000 \\
\hline QLD-N & 355 & Non-metallic mineral products & 0.000 & 0.000 \\
\hline QLD-N & 356 & Metals, metal products & -0.393 & 0.000 \\
\hline QLD-N & 357 & Machinery appliances and equipment & -0.130 & 0.000 \\
\hline QLD-N & 358 & Miscellaneous manufacturing & 0.000 & 0.000 \\
\hline QLD-N & 359 & Electricity supply, gas and water & -0.302 & 0.000 \\
\hline QLD-N & 360 & Residential building construction & -1.355 & -0.395 \\
\hline QLD-N & 361 & Other construction & -1.273 & -0.432 \\
\hline QLD-N & 362 & Trade & -2.009 & -1.040 \\
\hline QLD-N & 363 & Accommodation, cafes and restaurants & -1.330 & -0.185 \\
\hline QLD-N & 364 & Road transport & -1.287 & -0.303 \\
\hline QLD-N & 365 & Rail and pipeline transport & -0.310 & 0.000 \\
\hline QLD-N & 366 & Other transport & -0.391 & 0.000 \\
\hline QLD-N & 367 & Communication services & -0.013 & 0.000 \\
\hline QLD-N & 368 & Finance, property and business services & -0.955 & -0.242 \\
\hline QLD-N & 369 & Ownership of dwellings & 0.000 & 0.000 \\
\hline QLD-N & 370 & Government administration and defence & -0.292 & 0.000 \\
\hline QLD-N & 371 & Education & 0.000 & 0.000 \\
\hline QLD-N & 372 & Health and community services & 0.000 & 0.000 \\
\hline QLD-N & 373 & Cultural and recreational services & -0.641 & 0.000 \\
\hline QLD-N & 374 & Personal and other services & -0.036 & 0.000 \\
\hline QLD-FN & 375 & Sheep & 0.000 & 0.000 \\
\hline QLD-FN & 376 & Grains & 0.000 & 0.000 \\
\hline QLD-FN & 377 & Beef cattle & 0.000 & 0.000 \\
\hline QLD-FN & 378 & Dairy cattle and pigs & 0.000 & 0.000 \\
\hline QLD-FN & 379 & Other agriculture & -0.526 & 0.000 \\
\hline
\end{tabular}




\begin{tabular}{|c|c|c|c|c|}
\hline QLD-FN & 380 & Sugar cane growing & -0.006 & 0.000 \\
\hline QLD-FN & 381 & Forestry and fishing & 0.000 & 0.000 \\
\hline QLD-FN & 382 & Coal, oil and gas & 0.000 & 0.000 \\
\hline QLD-FN & 383 & Non-ferrous metal ores & 0.000 & 0.000 \\
\hline QLD-FN & 384 & Other mining & 0.000 & 0.000 \\
\hline QLD-FN & 385 & Food manufacturing & -0.037 & 0.000 \\
\hline QLD-FN & 386 & Textiles, clothing and footwear & 0.000 & 0.000 \\
\hline QLD-FN & 387 & Wood and paper manufacturing & 0.000 & 0.000 \\
\hline QLD-FN & 388 & Chemicals, petroleum and coal products & 0.000 & 0.000 \\
\hline QLD-FN & 389 & Non-metallic mineral products & 0.000 & 0.000 \\
\hline QLD-FN & 390 & Metals, metal products & 0.000 & 0.000 \\
\hline QLD-FN & 391 & Machinery appliances and equipment & 0.000 & 0.000 \\
\hline QLD-FN & 392 & Miscellaneous manufacturing & 0.000 & 0.000 \\
\hline QLD-FN & 393 & Electricity supply, gas and water & 0.000 & 0.000 \\
\hline QLD-FN & 394 & Residential building construction & 0.000 & 0.000 \\
\hline QLD-FN & 395 & Other construction & -0.327 & 0.000 \\
\hline QLD-FN & 396 & Trade & -0.826 & 0.000 \\
\hline QLD-FN & 397 & Accommodation, cafes and restaurants & -0.144 & 0.000 \\
\hline QLD-FN & 398 & Road transport & -0.151 & 0.000 \\
\hline QLD-FN & 399 & Rail and pipeline transport & 0.000 & 0.000 \\
\hline QLD-FN & 400 & Other transport & -0.358 & 0.000 \\
\hline QLD-FN & 401 & Communication services & 0.000 & 0.000 \\
\hline QLD-FN & 402 & Finance, property and business services & -0.732 & -0.019 \\
\hline QLD-FN & 403 & Ownership of dwellings & 0.000 & 0.000 \\
\hline QLD-FN & 404 & Government administration and defence & 0.000 & 0.000 \\
\hline QLD-FN & 405 & Education & 0.000 & 0.000 \\
\hline QLD-FN & 406 & Health and community services & 0.000 & 0.000 \\
\hline QLD-FN & 407 & Cultural and recreational services & 0.000 & 0.000 \\
\hline QLD-FN & 408 & Personal and other services & 0.000 & 0.000 \\
\hline QLD-NW & 409 & Sheep & 0.000 & 0.000 \\
\hline QLD-NW & 410 & Grains & 0.000 & 0.000 \\
\hline QLD-NW & 411 & Beef cattle & 0.000 & 0.000 \\
\hline QLD-NW & 412 & Dairy cattle and pigs & 0.000 & 0.000 \\
\hline QLD-NW & 413 & Other agriculture & 0.000 & 0.000 \\
\hline QLD-NW & 414 & Sugar cane growing & 0.000 & 0.000 \\
\hline QLD-NW & 415 & Forestry and fishing & 0.000 & 0.000 \\
\hline QLD-NW & 416 & Coal, oil and gas & 0.000 & 0.000 \\
\hline QLD-NW & 417 & Non-ferrous metal ores & -0.045 & 0.000 \\
\hline QLD-NW & 418 & Other mining & 0.000 & 0.000 \\
\hline QLD-NW & 419 & Food manufacturing & 0.000 & 0.000 \\
\hline QLD-NW & 420 & Textiles, clothing and footwear & 0.000 & 0.000 \\
\hline QLD-NW & 421 & Wood and paper manufacturing & 0.000 & 0.000 \\
\hline QLD-NW & 422 & Chemicals, petroleum and coal products & 0.000 & 0.000 \\
\hline
\end{tabular}




\begin{tabular}{|c|c|c|c|c|}
\hline QLD-NW & 423 & Non-metallic mineral products & 0.000 & 0.000 \\
\hline QLD-NW & 424 & Metals, metal products & 0.000 & 0.000 \\
\hline QLD-NW & 425 & Machinery appliances and equipment & 0.000 & 0.000 \\
\hline QLD-NW & 426 & Miscellaneous manufacturing & 0.000 & 0.000 \\
\hline QLD-NW & 427 & Electricity supply, gas and water & 0.000 & 0.000 \\
\hline QLD-NW & 428 & Residential building construction & 0.000 & 0.000 \\
\hline QLD-NW & 429 & Other construction & 0.000 & 0.000 \\
\hline QLD-NW & 430 & Trade & 0.000 & 0.000 \\
\hline QLD-NW & 431 & Accommodation, cafes and restaurants & 0.000 & 0.000 \\
\hline QLD-NW & 432 & Road transport & 0.000 & 0.000 \\
\hline QLD-NW & 433 & Rail and pipeline transport & 0.000 & 0.000 \\
\hline QLD-NW & 434 & Other transport & 0.000 & 0.000 \\
\hline QLD-NW & 435 & Communication services & 0.000 & 0.000 \\
\hline QLD-NW & 436 & Finance, property and business services & 0.000 & 0.000 \\
\hline QLD-NW & 437 & Ownership of dwellings & 0.000 & 0.000 \\
\hline QLD-NW & 438 & Government administration and defence & 0.000 & 0.000 \\
\hline QLD-NW & 439 & Education & 0.000 & 0.000 \\
\hline QLD-NW & 440 & Health and community services & 0.000 & 0.000 \\
\hline QLD-NW & 441 & Cultural and recreational services & 0.000 & 0.000 \\
\hline QLD-NW & 442 & Personal and other services & 0.000 & 0.000 \\
\hline SA & 443 & Sheep & -0.440 & 0.000 \\
\hline SA & 444 & Grains & -0.653 & 0.000 \\
\hline SA & 445 & Beef cattle & -0.481 & 0.000 \\
\hline SA & 446 & Dairy cattle and pigs & -0.046 & 0.000 \\
\hline SA & 447 & Other agriculture & -1.040 & -0.335 \\
\hline SA & 448 & Sugar cane growing & 0.000 & 0.000 \\
\hline SA & 449 & Forestry and fishing & -0.045 & 0.000 \\
\hline SA & 450 & Coal, oil and gas & -0.236 & -0.332 \\
\hline SA & 451 & Non-ferrous metal ores & -0.333 & 0.000 \\
\hline SA & 452 & Other mining & -0.677 & -0.406 \\
\hline SA & 453 & Food manufacturing & -1.093 & -0.293 \\
\hline SA & 454 & Textiles, clothing and footwear & 0.000 & 0.000 \\
\hline SA & 455 & Wood and paper manufacturing & -0.483 & 0.000 \\
\hline SA & 456 & Chemicals, petroleum and coal products & -0.636 & -0.040 \\
\hline SA & 457 & Non-metallic mineral products & -0.210 & 0.000 \\
\hline SA & 458 & Metals, metal products & -0.836 & -0.199 \\
\hline SA & 459 & Machinery appliances and equipment & -0.846 & 0.000 \\
\hline SA & 460 & Miscellaneous manufacturing & -0.370 & 0.000 \\
\hline SA & 461 & Electricity supply, gas and water & -0.882 & -0.357 \\
\hline SA & 462 & Residential building construction & -0.175 & 0.000 \\
\hline SA & 463 & Other construction & -1.122 & -0.280 \\
\hline SA & 464 & Trade & -1.671 & -0.702 \\
\hline SA & 465 & Accommodation, cafes and restaurants & -0.856 & 0.000 \\
\hline
\end{tabular}




\begin{tabular}{|c|c|c|c|c|}
\hline SA & 466 & Road transport & -1.060 & -0.076 \\
\hline SA & 467 & Rail and pipeline transport & -0.179 & 0.000 \\
\hline SA & 468 & Other transport & -0.924 & -0.280 \\
\hline SA & 469 & Communication services & -0.672 & -0.148 \\
\hline SA & 470 & Finance, property and business services & -1.707 & -0.994 \\
\hline SA & 471 & Ownership of dwellings & -0.331 & -0.403 \\
\hline SA & 472 & Government administration and defence & -0.763 & 0.000 \\
\hline SA & 473 & Education & -0.704 & 0.000 \\
\hline SA & 474 & Health and community services & 0.000 & 0.000 \\
\hline SA & 475 & Cultural and recreational services & -0.059 & 0.000 \\
\hline SA & 476 & Personal and other services & -0.629 & 0.000 \\
\hline WA & 477 & Sheep & -0.445 & 0.000 \\
\hline WA & 478 & Grains & -0.713 & -0.018 \\
\hline WA & 479 & Beef cattle & -0.582 & 0.000 \\
\hline WA & 480 & Dairy cattle and pigs & 0.000 & 0.000 \\
\hline WA & 481 & Other agriculture & -0.822 & -0.117 \\
\hline WA & 482 & Sugar cane growing & 0.000 & 0.000 \\
\hline WA & 483 & Forestry and fishing & -0.048 & 0.000 \\
\hline WA & 484 & Coal, oil and gas & -0.903 & -0.999 \\
\hline WA & 485 & Non-ferrous metal ores & -0.835 & -0.436 \\
\hline WA & 486 & Other mining & -1.734 & -1.463 \\
\hline WA & 487 & Food manufacturing & -0.920 & -0.120 \\
\hline WA & 488 & Textiles, clothing and footwear & -0.023 & 0.000 \\
\hline WA & 489 & Wood and paper manufacturing & -0.422 & 0.000 \\
\hline WA & 490 & Chemicals, petroleum and coal products & -0.892 & -0.296 \\
\hline WA & 491 & Non-metallic mineral products & -0.412 & 0.000 \\
\hline WA & 492 & Metals, metal products & -1.132 & -0.494 \\
\hline WA & 493 & Machinery appliances and equipment & -0.808 & 0.000 \\
\hline WA & 494 & Miscellaneous manufacturing & -0.487 & 0.000 \\
\hline WA & 495 & Electricity supply, gas and water & -0.983 & -0.458 \\
\hline WA & 496 & Residential building construction & -0.400 & 0.000 \\
\hline WA & 497 & Other construction & -1.474 & -0.633 \\
\hline WA & 498 & Trade & -1.840 & -0.870 \\
\hline WA & 499 & Accommodation, cafes and restaurants & -1.046 & 0.000 \\
\hline WA & 500 & Road transport & -1.247 & -0.263 \\
\hline WA & 501 & Rail and pipeline transport & -0.313 & 0.000 \\
\hline WA & 502 & Other transport & -1.191 & -0.547 \\
\hline WA & 503 & Communication services & -0.654 & -0.131 \\
\hline WA & 504 & Finance, property and business services & -1.930 & -1.217 \\
\hline WA & 505 & Ownership of dwellings & -0.622 & -0.695 \\
\hline WA & 506 & Government administration and defence & -0.874 & 0.000 \\
\hline WA & 507 & Education & -0.898 & 0.000 \\
\hline WA & 508 & Health and community services & -0.059 & 0.000 \\
\hline
\end{tabular}




\begin{tabular}{|c|c|c|c|c|}
\hline WA & 509 & Cultural and recreational services & -0.256 & 0.000 \\
\hline WA & 510 & Personal and other services & -0.786 & 0.000 \\
\hline TAS & 511 & Sheep & 0.000 & 0.000 \\
\hline TAS & 512 & Grains & 0.000 & 0.000 \\
\hline TAS & 513 & Beef cattle & -0.001 & 0.000 \\
\hline TAS & 514 & Dairy cattle and pigs & 0.000 & 0.000 \\
\hline TAS & 515 & Other agriculture & -0.436 & 0.000 \\
\hline TAS & 516 & Sugar cane growing & 0.000 & 0.000 \\
\hline TAS & 517 & Forestry and fishing & -0.168 & 0.000 \\
\hline TAS & 518 & Coal, oil and gas & 0.000 & 0.000 \\
\hline TAS & 519 & Non-ferrous metal ores & 0.000 & 0.000 \\
\hline TAS & 520 & Other mining & -0.230 & 0.000 \\
\hline TAS & 521 & Food manufacturing & -0.526 & 0.000 \\
\hline TAS & 522 & Textiles, clothing and footwear & 0.000 & 0.000 \\
\hline TAS & 523 & Wood and paper manufacturing & -0.014 & 0.000 \\
\hline TAS & 524 & Chemicals, petroleum and coal products & 0.000 & 0.000 \\
\hline TAS & 525 & Non-metallic mineral products & 0.000 & 0.000 \\
\hline TAS & 526 & Metals, metal products & -0.268 & 0.000 \\
\hline TAS & 527 & Machinery appliances and equipment & 0.000 & 0.000 \\
\hline TAS & 528 & Miscellaneous manufacturing & 0.000 & 0.000 \\
\hline TAS & 529 & Electricity supply, gas and water & -0.357 & 0.000 \\
\hline TAS & 530 & Residential building construction & 0.000 & 0.000 \\
\hline TAS & 531 & Other construction & -0.535 & 0.000 \\
\hline TAS & 532 & Trade & -1.112 & -0.142 \\
\hline TAS & 533 & Accommodation, cafes and restaurants & -0.311 & 0.000 \\
\hline TAS & 534 & Road transport & -0.484 & 0.000 \\
\hline TAS & 535 & Rail and pipeline transport & 0.000 & 0.000 \\
\hline TAS & 536 & Other transport & -0.383 & 0.000 \\
\hline TAS & 537 & Communication services & -0.109 & 0.000 \\
\hline TAS & 538 & Finance, property and business services & -1.038 & -0.325 \\
\hline TAS & 539 & Ownership of dwellings & 0.000 & 0.000 \\
\hline TAS & 540 & Government administration and defence & -0.272 & 0.000 \\
\hline TAS & 541 & Education & -0.138 & 0.000 \\
\hline TAS & 542 & Health and community services & 0.000 & 0.000 \\
\hline TAS & 543 & Cultural and recreational services & 0.000 & 0.000 \\
\hline TAS & 544 & Personal and other services & -0.035 & 0.000 \\
\hline ACT & 545 & Sheep & 0.000 & 0.000 \\
\hline ACT & 546 & Grains & 0.000 & 0.000 \\
\hline ACT & 547 & Beef cattle & 0.000 & 0.000 \\
\hline ACT & 548 & Dairy cattle and pigs & 0.000 & 0.000 \\
\hline ACT & 549 & Other agriculture & 0.000 & 0.000 \\
\hline ACT & 550 & Sugar cane growing & 0.000 & 0.000 \\
\hline ACT & 551 & Forestry and fishing & 0.000 & 0.000 \\
\hline
\end{tabular}




\begin{tabular}{|c|c|c|c|c|}
\hline ACT & 552 & Coal, oil and gas & 0.000 & 0.000 \\
\hline ACT & 553 & Non-ferrous metal ores & 0.000 & 0.000 \\
\hline ACT & 554 & Other mining & 0.000 & 0.000 \\
\hline ACT & 555 & Food manufacturing & 0.000 & 0.000 \\
\hline ACT & 556 & Textiles, clothing and footwear & 0.000 & 0.000 \\
\hline ACT & 557 & Wood and paper manufacturing & 0.000 & 0.000 \\
\hline ACT & 558 & Chemicals, petroleum and coal products & 0.000 & 0.000 \\
\hline ACT & 559 & Non-metallic mineral products & 0.000 & 0.000 \\
\hline ACT & 560 & Metals, metal products & 0.000 & 0.000 \\
\hline ACT & 561 & Machinery appliances and equipment & 0.000 & 0.000 \\
\hline ACT & 562 & Miscellaneous manufacturing & 0.000 & 0.000 \\
\hline ACT & 563 & Electricity supply, gas and water & 0.000 & 0.000 \\
\hline ACT & 564 & Residential building construction & 0.000 & 0.000 \\
\hline ACT & 565 & Other construction & -0.382 & 0.000 \\
\hline ACT & 566 & Trade & -0.828 & 0.000 \\
\hline ACT & 567 & Accommodation, cafes and restaurants & -0.192 & 0.000 \\
\hline ACT & 568 & Road transport & -0.144 & 0.000 \\
\hline ACT & 569 & Rail and pipeline transport & 0.000 & 0.000 \\
\hline ACT & 570 & Other transport & -0.178 & 0.000 \\
\hline ACT & 571 & Communication services & -0.173 & 0.000 \\
\hline ACT & 572 & Finance, property and business services & -1.183 & -0.470 \\
\hline ACT & 573 & Ownership of dwellings & 0.000 & 0.000 \\
\hline ACT & 574 & Government administration and defence & -0.772 & 0.000 \\
\hline ACT & 575 & Education & -0.102 & 0.000 \\
\hline ACT & 576 & Health and community services & 0.000 & 0.000 \\
\hline ACT & 577 & Cultural and recreational services & 0.000 & 0.000 \\
\hline ACT & 578 & Personal and other services & -0.006 & 0.000 \\
\hline NT & 579 & Sheep & 0.000 & 0.000 \\
\hline NT & 580 & Grains & 0.000 & 0.000 \\
\hline NT & 581 & Beef cattle & 0.000 & 0.000 \\
\hline NT & 582 & Dairy cattle and pigs & 0.000 & 0.000 \\
\hline NT & 583 & Other agriculture & 0.000 & 0.000 \\
\hline NT & 584 & Sugar cane growing & 0.000 & 0.000 \\
\hline NT & 585 & Forestry and fishing & 0.000 & 0.000 \\
\hline NT & 586 & Coal, oil and gas & 0.000 & 0.000 \\
\hline NT & 587 & Non-ferrous metal ores & 0.000 & 0.000 \\
\hline NT & 588 & Other mining & -0.026 & 0.000 \\
\hline NT & 589 & Food manufacturing & 0.000 & 0.000 \\
\hline NT & 590 & Textiles, clothing and footwear & 0.000 & 0.000 \\
\hline NT & 591 & Wood and paper manufacturing & 0.000 & 0.000 \\
\hline NT & 592 & Chemicals, petroleum and coal products & 0.000 & 0.000 \\
\hline NT & 593 & Non-metallic mineral products & 0.000 & 0.000 \\
\hline NT & 594 & Metals, metal products & 0.000 & 0.000 \\
\hline
\end{tabular}




\begin{tabular}{|l|c|l|c|c|}
\hline NT & 595 & Machinery appliances and equipment & 0.000 & 0.000 \\
\hline NT & 596 & Miscellaneous manufacturing & 0.000 & 0.000 \\
\hline NT & 597 & Electricity supply, gas and water & 0.000 & 0.000 \\
\hline NT & 598 & Residential building construction & 0.000 & 0.000 \\
\hline NT & 599 & Other construction & -0.244 & 0.000 \\
\hline NT & 600 & Trade & -0.650 & 0.000 \\
\hline NT & 601 & Accommodation, cafes and restaurants & 0.000 & 0.000 \\
\hline NT & 602 & Road transport & -0.068 & 0.000 \\
\hline NT & 603 & Rail and pipeline transport & 0.000 & 0.000 \\
\hline NT & 604 & Other transport & -0.198 & 0.000 \\
\hline NT & 605 & Communication services & 0.000 & 0.000 \\
\hline NT & 606 & Finance, property and business services & -0.691 & 0.000 \\
\hline NT & 607 & Ownership of dwellings & 0.000 & 0.000 \\
\hline NT & 608 & Government administration and defence & -0.277 & 0.000 \\
\hline NT & 609 & Education & 0.000 & 0.000 \\
\hline NT & 610 & Health and community services & 0.000 & 0.000 \\
\hline NT & 611 & Cultural and recreational services & 0.000 & 0.000 \\
\hline NT & 612 & Personal and other services & 0.000 & 0.000 \\
\hline ROW & 613 & ROW & 0.000 & 0.000 \\
\hline
\end{tabular}




\section{SI 5: References}

Agence-France-Presse: Debbie's devastation: First victims of ferocious storm tell of damage. In: Agence-FrancePresse, 28 March, 2017.

Australian Government: Budget Strategy and Outlook 2011-12. Canberra, 2011.

BBC News: Cyclone Debbie: Thousands evacuate in Queensland, Australia. In: BBC News, 2017.

Carruthers, P.: Eight council areas get disaster relief due to Cyclone Debbie. In: The Morning Bulletin, 2017.

Courier Mail, 2017.

Cummins, A.: Queensland cyclone Debbie prompts evacuations in Australia. In: WFMZ-TV, 2017.

Department of the Prime Minister and Cabinet: Best Practice Regulation Guidance Note Value of statistical life.

Canberra, 2014.

Donoughue, P.: Cyclone Debbie nothern NSW braces for major flooding. In: ABC News, 2017.

Emerick, C.: Cyclone Debbie caused over $\$ 100 \mathrm{~m}$ in damages for Australian growers In: Fresh Plaza, 2017.

Flaherty, J.: Cyclone Debbie - Overall Damage Bill and Economic Impact. In: CCIQ, 2017.

Gillman, 0.: Debbie's disastrous aftermath: Incredible pictures show the billion-dollar clean up underway after Cyclone Debbie ravaged Australia's east coast - as devastated residents arrive home. In: Daily Mail Australia, 2017.

Cyclone Debbie aftermath: Business owners turn on Malcolm Turnbull as he tours flood ravaged Lismore, Available online: http://www.dailytelegraph.com.au/business/cyclone-debbie-aftermath-business-owners-turn-on-malcolmturnbull-as-he-tours-flood-ravaged-lismore/news-story/b09f194fa876d0cabd623889172cedc6:

http://www.dailytelegraph.com.au/business/cyclone-debbie-aftermath-business-owners-turn-on-malcolm-

turnbull-as-he-tours-flood-ravaged-lismore/news-story/b09f194fa876d0cabd623889172cedc6, access: 15 June, April 3, 2017 1:36pm.

Lismore floods: Business owners without insurance hit hardest, could be forced to close forever, Available online: http://www.dailytelegraph.com.au/news/nsw/lismore-floods-business-owners-without-insurance-hit-hardestcould-be-forced-to-close-forever/news-story/51a52a4f328436cb3790defd2ce8280d:

http://www.dailytelegraph.com.au/business/cyclone-debbie-aftermath-business-owners-turn-on-malcolmturnbull-as-he-tours-flood-ravaged-lismore/news-story/b09f194fa876d0cabd623889172cedc6, access: 15 June, April 9, 2017 12:00am.

Honan, K.: Flood-affected farmers in New South Wales call for disaster funding three weeks on. In: ABC News, 2017.

John McCarthy, and Vogler, S.: Queensland. Cyclone Debbie economic impact. In: Courier Mail, 2017.

Killalea, D., Reynolds, E., and Palin, M.: Grim warning as Debbie bears down: 'This is a monster cyclone'. In: NewsComAu, 2017.

Neville, M.: Cyclone Debbie's \$6 million impact on dairy. In: QDO, 12 April, 2017.

North Queensland Bulk Ports Corporation: NQBP ports prepared forTropical Cyclone Debbie, 2017.

O'Brien, C.: Cyclone Debbie recovery costs to hit Queensland budget bottom line. In: ABC News, 2017.

Public-Private Partnership in Infrastructure Resource Centre: Central Queensland Coal Network - Model Operator Access Agreement Coal, World Bank Group, 2016.

QFF: Cyclone Debbie Agricultural Impact - 6 April 2017. 2017.

Queensland Government: The State Recovery Plan 2017-2019. Operation Queensland Recovery. Working to recover, reconnect and rebuild more resilient Queensland communities following the effects of Severe Tropical Cyclone Debbie. Queensland Government, Brisbane, 2017.

Robertson, J.: Cyclone Debbie: Queensland reels at the scale of destruction In: The Guardian, Australia, 2017.

Serapio, M.: With Australia's supply disrupted by Cyclone Debbie, coal buyers race elsewhere. In: Sydney Morning Herald, 2017.

The Courier Mail: Queensland cyclone: Debbie damage to cost local sugar industry $\$ 150$ million. In: Courier Mail, 2017.

Tourism Research Australia: Tourism Businesses in Australia, June 2011 to June 2015, Tourism Research Australia, Canberra, 2016.

Underwriter, C.: Insured losses from Cyclone Debbie reach AU\$306 million: Insurance Council of Australia In: Canadian Underwriter, 4 April 2017.

Young, M., Chang, C., Reynolds, E., and Craw, V.: Cyclone Debbie's full wrath is revealed. In: NewsComAu, 2017. 